\title{
Coordinating Feet in Bipedal Balance
}

\author{
S.O. Anderson, C.G. Atkeson, J.K. Hodgins \\ Robotics Institute \\ Carnegie Mellon University \\ soa,cga,jkh@ri.cmu.edu
}

\begin{abstract}
Biomechanical models of human standing balance in the sagittal plane typically treat the two ankle joints as a single degree of freedom. They describe the sum of the torques produced by the ankles, but do not predict what the contribution of each ankle will be. Similarly, balance algorithms for bipedal robots control the location of the overall center of pressure, but do not consider the individual centers of pressure under each foot. We present theoretical and experimental results showing an optimal solution to the problem of producing a single desired torque with redundant actuators, resulting in alignment of the individual centers of pressure under each foot. This produces a feedback gain structure not addressed in the biomechanics literature and a balance controller that is potentially more robust to unexpected changes in the region of support. We show that the feedback gain matrix of this controller has an unexpected structure - large off-axis integral gain elements indicate that the ankle torque that equalize the position of the center of pressure is determined primarily by information from the other foot. We also demonstrate controllers based on this design using the Sarcos Primus hydraulic biped.
\end{abstract}

\section{INTRODUCTION}

Models of human balance during stance typically take the form of an inverted pendulum with one or more links [1], [2]. Torques about some of these links represent the combined action of two joints or actuators, for example, the left and right ankles, but do not describe the individual contribution of each actuator. Similarly, many balance algorithms control the center of pressure for the whole system, but are not concerned with equalizing the individual centers of pressure under each foot [3], [4]. Controlling individual centers of pressure can improve efficiency during balance and stability during changes in contact state.

In robotics, control of total ground reaction force, typically through control of the zero moment point (ZMP), is the objective of most balance algorithms [5], [6], [7]. For any given location of the ZMP, there is a space of possible configurations for the individual centers of pressure under each foot. While the ZMP criterion is sufficient to maximize stability given the current set of contact points, control of the individual centers of pressure is important for maintaining stable contact of each foot and overall stability during changes in contact state.

Balance in the presence of unexpected changes to the region of support can be improved if the individual centers of pressure are simultaneously controlled to be in the middle of the contact region of each foot. If the individual centers of pressure move toward the edges of the feet, the ZMP remains unaffected, but it becomes more likely that one of the feet will

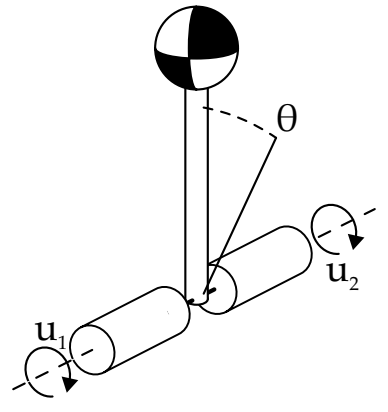

Fig. 1. Dual actuator model used in simulations

lose contact with the ground. Additionally, if one foot loses contact with the ground then the remaining ankle will lose its counterbalancing torque and the ZMP will quickly move away from the center of the contact region. Finally, having an uneven pressure distribution under the feet can reduce the size of the contact region as a compliant floor conforms to the feet, reducing the maximum possible reactive excursion of the ZMP in response to perturbations. These potential failures indicate that controlling the individual centers of pressure in the nullspace of the combined center of pressure's motion can improve stability.

We present a novel model of quiet stance that includes the individual centers of pressure under each foot. We then use this model to show that two integrator states are required in order for the model to equalize the individual centers of pressure under each foot when an optimal symmetric linear PID controller is used. Both simulation and experimental results show that no controller design with a single integrator state is capable of eliminating steady-state error from the system. Additionally, we show that optimal feedback gains for each actuator place a higher weight on the integrator state driven by the contralateral actuator than on the integrator state driven by the actuator itself.

Section II of this paper describes the model of the balancing robot and the structure of the optimal PID feedback controller for this model. Section III describes our numerical simulations of this model. These experiments demonstrate the necessity of the control structure derived in Section II. Section IV presents a validation of the simulation results obtained by implementing the simulated controllers on the Sarcos Primus hydraulic biped and comparing the robot's behavior to that of the simulator. Section $\mathrm{V}$ builds on these results with further discussion of the model and its implications. 


\section{MODEL}

The model is the dual actuator single link inverted pendulum illustrated in Fig. 1. The system state vector has four components:

$$
\mathbf{x}_{t}=\left\{\theta, \dot{\theta}, a_{1}, a_{2}\right\}^{\top}
$$

$\theta$ and $\dot{\theta}$ are the body angle and body angular velocity, respectively, and $a_{1}$ and $a_{2}$ accumulate the total torque applied by each actuator and function as integrators for a PID controller. By linearizing the system about vertical stance, the dynamics including a linear controller $\mathbf{u}=-\mathbf{K} \mathbf{x}_{t}$ can be described by the following state update equation:

$$
\begin{aligned}
\mathbf{x}_{t+1} & =(\mathbf{A}-\mathbf{B K}) \mathbf{x}_{t} \\
\mathbf{A} & =\left(\begin{array}{cccc}
1 & t_{s} & 0 & 0 \\
\frac{m l g}{I} t_{s} & 1 & 0 & 0 \\
0 & 0 & 1 & 0 \\
0 & 0 & 0 & 1
\end{array}\right) \\
\mathbf{B} & =\left(\begin{array}{cc}
0 & 0 \\
\frac{1}{I} t_{s} & \frac{1}{I} t_{s} \\
t_{s} & 0 \\
0 & t_{s}
\end{array}\right)
\end{aligned}
$$

where $m$ and $l$ are the mass and length of the pendulum, $I$ is the rotational inertia about the joint, $g$ is acceleration due to gravity, and $t_{s}$ is the time step. This model does not address the influence of foot size on the behavior of the system, particularly the potential for a foot to lift off from the ground if a large torque is applied. We assume, and our simulations reflect, that the applied torques are sufficiently small that ten centimeter long feet would not lift off the ground.

A linear quadratic regulator (LQR)[8] design procedure yields a feedback gain matrix that simultaneously minimizes the difference between the applied torques, the total applied torque, body sway, and body velocity. When optimal LQR control gains are computed for this system the gain matrix $\mathbf{K}$ has the following structure:

$$
\mathbf{K}=\left(\begin{array}{llll}
P & D & I_{1} & I_{2} \\
P & D & I_{2} & I_{1}
\end{array}\right)
$$

The LQR solution for this system, given a reasonable time step, has $\left|I_{1}\right| \ll\left|I_{2}\right|$. This structure is significant because it indicates that the torque output from the actuators are crosscoupled, that is, each actuator is influenced more by the integrator driven by the torque delivered by the other foot than by the integrator driven by the torque on its foot.

This structure also follows directly from an inspection of the model. Because the two torque sources and integrator states are symmetric, the corresponding terms in $K$ must be equal. Furthermore, the sign of the $I_{2}$ term can be determined by considering how the behavior of the whole system will change as the sign of that term changes. The state update equation yields the following equations:

$$
\begin{aligned}
a_{1_{n+1}}-a_{2_{n+1}} & =\left(a_{1_{n}}-a_{2_{n}}\right)\left(1+t_{S}\left(I_{2}-I_{1}\right)\right) \\
u_{1}-u_{2} & =\left(a_{1_{n}}-a_{2_{n}}\right)\left(I_{2}-I_{1}\right)
\end{aligned}
$$

These equations indicate that the difference between the integrator states determines the difference between the torques, and that the change in both of the differences over time depends on the values $I_{1}$ and $I_{2}$. If the magnitude of the difference between the actuator torques is to decrease then it must be the case that $-2<t_{s}\left(I_{2}-I_{1}\right)<0$. Typically, integrator gains have negative sign - a positive gain causes the system to comply with steady-state disturbances rather than compensate for them. Suppose $I_{2}$ were non-negative and the system was stable. Equation (6) above indicates that $\left|a_{1}-a_{2}\right|$ does not decrease unless $I_{1}$ is larger than $I_{2}$. However, if $I_{2}>I_{1}$ then the behavior of the system when $a_{1}=a_{2}$ will be unstable. Specifically, the system will behave identically to a single actuator model with:

$$
\mathbf{K}_{u}=2\left(\begin{array}{lll}
P & D & \left(I_{1}+I_{2}\right)
\end{array}\right)
$$

$\mathbf{K}_{u}$ is unstable because $I_{1}+I_{2}>0$. Therefore, in order for the system to be stable and bring the actuator torques to the same value, $I_{2}$ must have a larger magnitude than $I_{1}$ and be negative. The optimal feedback gains for the model must therefore have a cross-coupled structure.

\section{Simulation}

We explored the behavior of this model using numerical simulations to compare the behavior of four control designs. Two of these designs use only a single integrator state. The first design integrates only the sum of the applied torques. while the second integrates only the difference of the applied torques. The remaining two designs contain two integrator states. The first of these combines two identical and independent controllers each designed to control half the mass of the system, the second allows full feedback between the integrator states. The simulation results shown in Fig. 2 demonstrate the different failure modes for the first three controllers and the success of the full state feedback controller. The simulation used parameters that match the Sarcos Primus. (Table I.)

TABLE I

SiMULATION PARAMETERS

\begin{tabular}{|l|c|c|}
\hline mass $\times$ length & $m l$ & $34.29 \mathrm{kgm}^{2}$ \\
\hline rotational inertia & $I$ & $52.39 \mathrm{kgm}^{2}$ \\
\hline gravitational acceleration & $g$ & $9.81 \mathrm{~ms}^{-2}$ \\
\hline time step & $t_{s}$ & $0.001 \mathrm{~s}$ \\
\hline
\end{tabular}

All the LQR feedback gains were designed using identity matrices for the $\mathbf{Q}$ and $\mathbf{R}$ cost matrices. The simulated right foot actuator produced an additional torque of $-0.5 \mathrm{Nm}$ to simulate a miscalibration in the right foot force controller

Case 1: The first controller uses LQR feedback gains designed for a single actuator with one torque integrator state containing the sum of the applied torques. The desired torque produced by these gains is divided equally between the two actuators. The integrator accumulates the sum of the torques applied by the actuators. The simulation results from this controller show that while the joint center of pressure quickly 

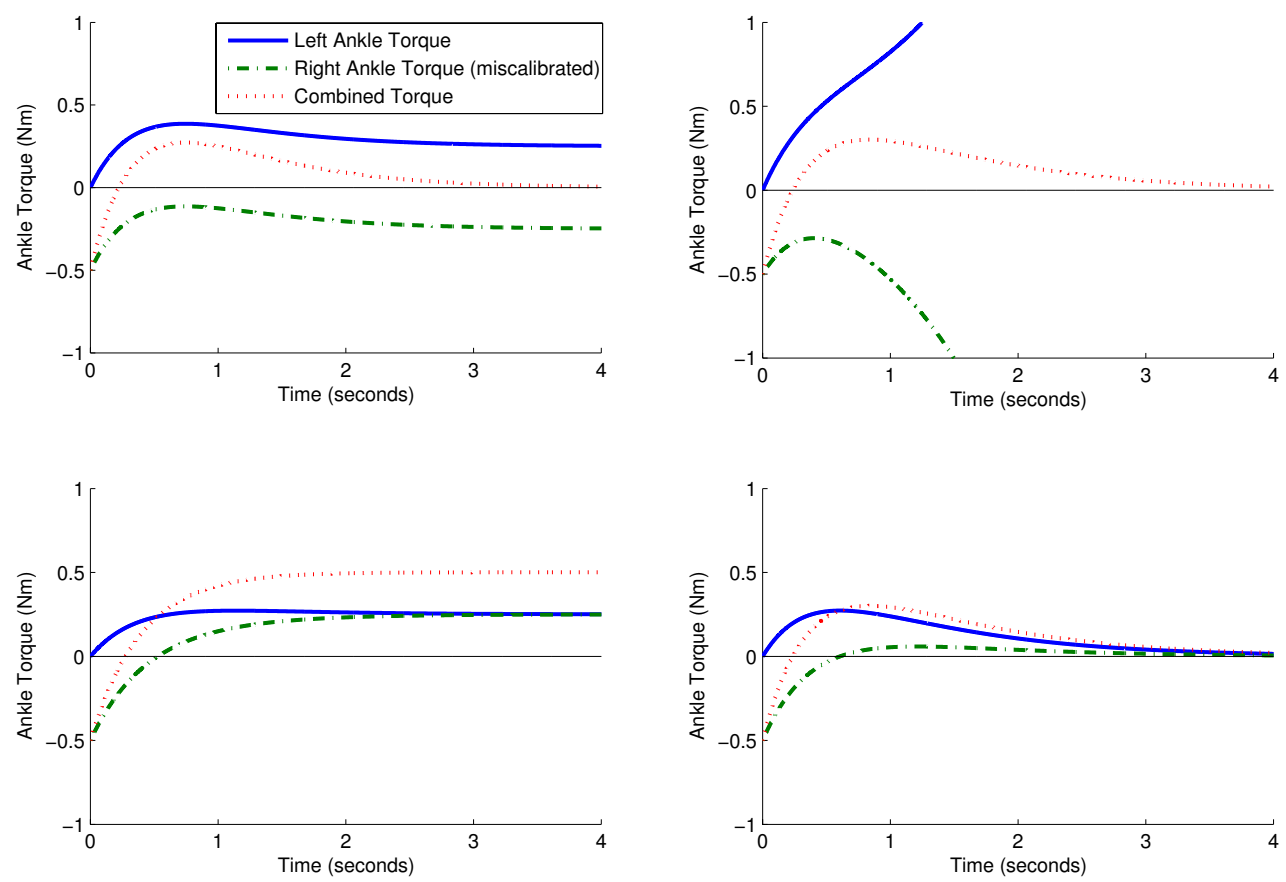

Fig. 2. Numerical simulations of LQR controllers: Simulation of LQR gains designed for a single integrator for the sum of applied torques (top left). Single integrator for the difference between applied torques (bottom left). Two integrators without cross coupling (top right). Two integrators with cross coupling (bottom right)
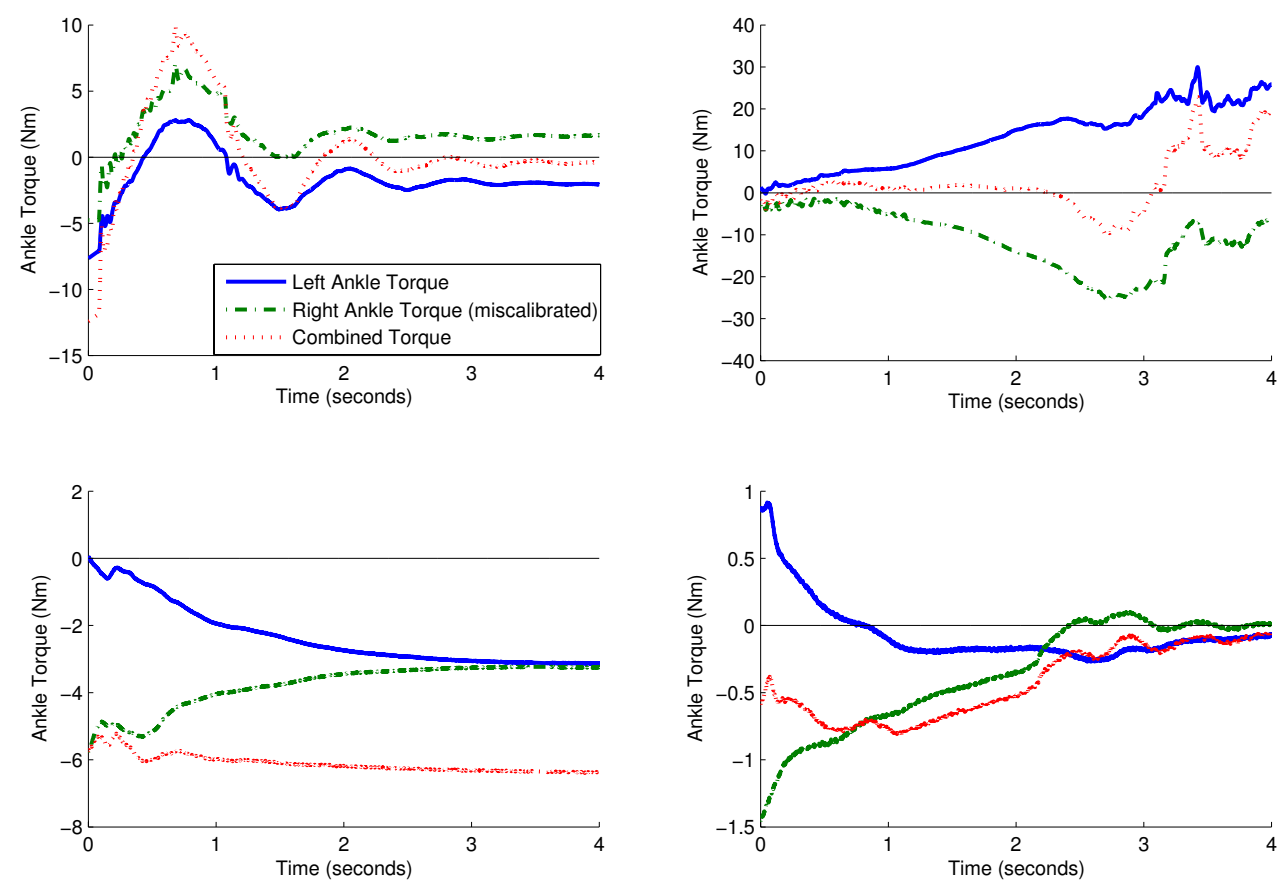

Fig. 3. Robot experiments using gains based on those derived for the LQR simulations in Fig. 2. Single integrator for the sum of applied torques (top left). Single integrator for the difference of the applied torques (bottom left). Decoupled double integrators (top right). Coupled double integrators (bottom right). 
goes to zero, there is a steady-state offset between the left and right foot center of pressure.

Case 2: The second controller design integrates the difference between the applied torques, rather than their sum. This system was designed with optimal LQR gains for a dual actuator system with a single integrator state. While this system is stable in the presence of uncorrelated disturbances, it is not able to compensate for the calibration error. This defect can be seen in the steady-state offset of the combined center of pressure. This design and the previous control design are the only possible symmetric linear PID control designs, demonstrating that more than one integrator is necessary to stabilize the system and equalize center of pressure locations.

Case 3: The third simulation uses LQR gains based on a single actuator model with half the mass of the simulated model. The full system is simulated by creating two integrator states and applying these feedback gains independently to each actuator. The joint center of pressure goes to zero while the individual centers of pressure diverge in opposite directions. Because the body dynamics are sensitive only to the sum of the torques about the ankles, the simulated body angle and velocity are stable in this simulation, despite the large individual torque magnitudes.

Case 4: The final design, using a matrix with cross-coupled gain structure, produces the desired behavior. This simulation includes the dual integrators in the LQR design procedure. The simulation shows that despite the miscalibration in the right ankle actuator the system is able to both stabilize the body and bring the individual centers of pressure into alignment. The optimal LQR gains for the model parameters as chosen are

$$
\mathbf{K}_{\text {sim }}=\left(\begin{array}{cccc}
468 & 185 & 0.001 & -0.998 \\
468 & 185 & -0.998 & 0.001
\end{array}\right)
$$

\section{ROBOT EXPERIMENTS}

This section describes the results of implementing the feedback controllers discussed in section III on the Sarcos Primus hydraulic robot (Fig. 4). Some gains had to be scaled in order to stabilize the robot, possibly because of discrepancies between the linear model and the real robot. Gains used on the robot in case 4 are shown below. As in the simulation, an offset torque was added to the commanded value for the right ankle actuator.

$$
\mathbf{K}_{\text {robot }}=\left(\begin{array}{cccc}
468 & 1 & 0.001 & -0.998 \\
468 & 1 & -0.998 & 0.001
\end{array}\right)
$$

The results in Fig. 3 show that the robot produced similar behavior to that predicted by the simulations in all four scenarios. When a single integrator storing the sum of the ankle torques was used (case 1), the combined torque had no steady-state error, but there was a steady-state offset between the joint torques, much like the behavior we saw in the simulator. Likewise, when a single integrator storing the difference between ankle torques was used (case 2), the difference between the torques went to zero, but there was a steady-state error in the combined torque, as in the simulation.

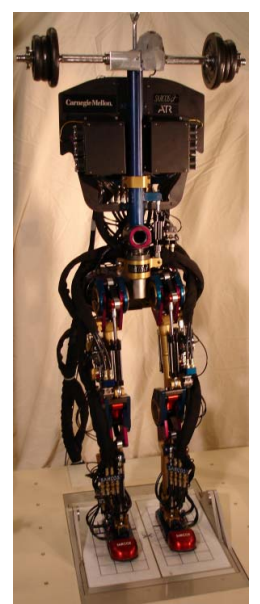

Fig. 4. Sarcos Primus hydraulic biped

When a separate PID controller was used on each ankle with gains designed to stabilize half the mass of the pendulum (case 3), the combined torque stabilized the system, but the individual torques grew quickly. This growth caused the left foot to rock back on the heel while the right foot rocked forward onto the toe as in the simulation. The cross-coupled gains (case 4) produced stable behavior that brought both the combined torque and the difference between the torques to zero.

\section{DISCUSSION}

It is not surprising that two state variables are required to control steady-state error, as there are two degrees of freedom, each of which can have a steady-state error, in the dual actuator model. Steady-state differences between the applied torques and steady-state offsets in their sum are independent degrees of freedom in the model. It is surprising that the cross coupling gains are much larger than the direct gains. The cross-coupled gain structure is not a result of the system model being nonminimum phase. If $\mathbf{A}$ is replaced with the identity matrix (a minimum phase system) and $\mathbf{B}$ is truncated such that:

$$
\mathbf{B}=\left(\begin{array}{ll}
1 & 1 \\
1 & 0 \\
0 & 1
\end{array}\right)
$$

the $\mathrm{LQR}$ procedure still produces an optimal gain matrix with a cross-coupled structure.

The B matrix for the dual actuator model can also be rewritten to yield an alternate structure for $\mathbf{K}$ :

$$
\begin{aligned}
\mathbf{B}_{a} & =\left(\begin{array}{cc}
0 & 0 \\
1 & 1 \\
1 & 1 \\
1 & -1
\end{array}\right) \\
\mathbf{K}_{a} & =\left(\begin{array}{cccc}
P & D & I_{1} & I_{2} \\
P & D & I_{1} & -I_{2}
\end{array}\right)
\end{aligned}
$$

where $I_{1}>0$ and $I_{2}>0$. In this formulation the third system state corresponds to the integrated sum of the torque 


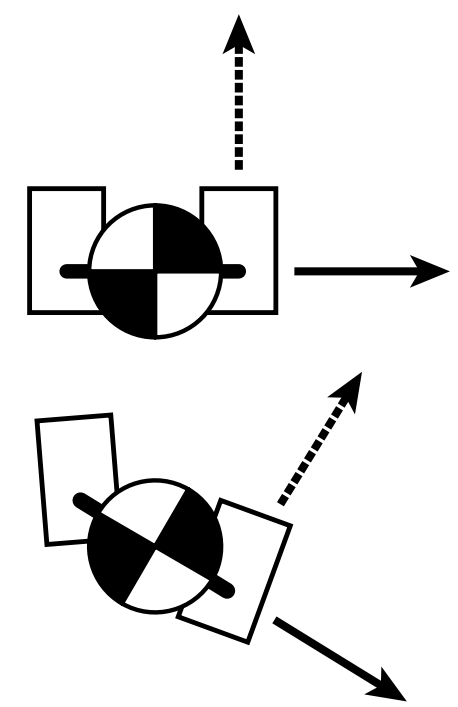

Fig. 5. Typical foot placement during quiet stance as assumed in our model, solid arrow indicates axis about which ankle torque are cumulative, dashed arrow indicates axis about which ankle torques do not affect the motion of the body(top). Alternate foot placement, however, the ankle torques are still cumulative about the solid arrow(bottom).

and the fourth state corresponds to the integrated difference. This structure allows the $\mathbf{Q}$ matrix to independently penalize differences between the applied torques and the sum of the torques. Additionally, $I_{1}$ is equal to the value derived for a model that includes only the integration of summed torques and $I_{2}$ is equal to the value derived for a model that includes only the integration of torque differences, given identical $\mathbf{Q}$ and $\mathbf{R}$ matrices.

PID control of other symmetric degrees of freedom does not necessarily exhibit cross-coupled gain structure. For example, in our model, the ankle torques in the mediolateral plane have distinct effects on the body dynamics - that is, they do not sum in the same fashion that torques in the sagittal plane do. This decoupling occurs because the rotational axes of the abduct/adduct joints are distinct, whereas the ankles share a single rotational axis in the sagittal plane. Because the ankles do not share a rotational axis, torque in the mediolateral plane about one ankle produces only vertical force on the other foot. This observation generalizes to include arbitrary foot placements on uneven terrain. In general, the vector between the ankles defines the axis about which rotation is free to occur, and about which ankle torques couple as described in our model. The axis perpendicular to that vector defines the axis around which the system is passively stable, and about which ankle torques do not couple. Fig. 5 illustrates this concept.

Bipeds are unique among legged balancers in that it is always the case that more than one ankle is able to apply torque on an axis about which rotational motion is not passively stable. Modeling balance with only a single foot excludes the possibility of redundant actuation, as there is only one torque source. Balance with three or more feet permits passive stability when the center of mass is above the convex hull of the ankles. If the vertical projection of the center of mass lies outside the convex hull of the ankles, then only the ankle joints lying along the edge of the hull closest to the center of mass need be active. Except in the degenerate case of three or more collinear ankles this situation reduces to the single or double foot case. In the case of three or more collinear ankles the model presented earlier can be extended to include three integrator states, and the feedback gains indicate strong coupling between the state of all three symmetric collinear actuators.

Future work will include experiments with human subjects to test for the presence of this integral gain structure in two-legged balance. We plan to modify a clinical balance testing platform so that it can apply a disturbance to only one foot while recording the torque response of both feet. The experimental transfer functions gathered from human subjects can then be compared with those predicted by the model and obtained from the Sarcos Primus to determine if human balance exhibits a cross-coupled integral gain structure. We also believe that individual control of the centers of pressure can be used to decrease sensitivity to unexpected perturbations in the dual stance phase of bipedal gait.

\section{ACKNOWLEDGMENT}

This material is based upon work supported in part by the National Science Foundation under NSF Grants CNS0224419, DGE-0333420, and ECS-0325383.

\section{REFERENCES}

[1] R. J. Peterka, "Sensorimotor integration in human postural control," Journal of Neurophysiology, vol. 88, pp. 1097-1118, 2002.

[2] H. van der Kooij, B. Koopman, and F. van der Helm, "An adaptive model of sensory integration in a dynamic environment applied to human stance control," Biological Cybernetics, vol. 84, no. 2, pp. 103-115, 2001.

[3] W. L. Wooten, "Simulation of leaping, tumbling, landing, and balancing humans," Ph.D. dissertation, Georgia Tech, 1998

[4] M. Vukobratovic, "How to control the artificial anthropomorphic systems," IEEE Trans. System, Man and Cybernetics SMC-3, pp. 497-507, 1973.

[5] K. I. S. Kudoh, T. Komura, "The dynamic postural adjustment with the quadratic programming method," in Proc., Int. Conf. on Intelligent Robots and Systems, 2002, pp. 2563-2568.

[6] S. Ita, T. Nishigaki, and H. Kawasaki, "Upright posture stabilization by ground reaction force control," in Proc., Int. Sympo. On Measurement, Analysis and Modeling of Human Functions (ISHF2001), 2001, pp. 515520

[7] S. Kajita, K. Yokio, M. Saigo, and K. Tanie, "Balancing a humanoid robot using backdrive concerned torque control and direct angular momentum feedback," in Proc., IEEE Int. Conf. on Robotics and Automation, vol. 4. IEEE, 2001, pp. 3376-3382.

[8] R. F. Stengel, Optimal Control and Estimation. New York: Dover, 1994. 